NISSUNA UMANA INVESTIGAZIONE SI PUO DIMANDARE VERA SCIENZIA S'ESSA NON PASSA PER LE MATEMATICHE DIMOSTRAZIONI LEONARDO DA VINCI

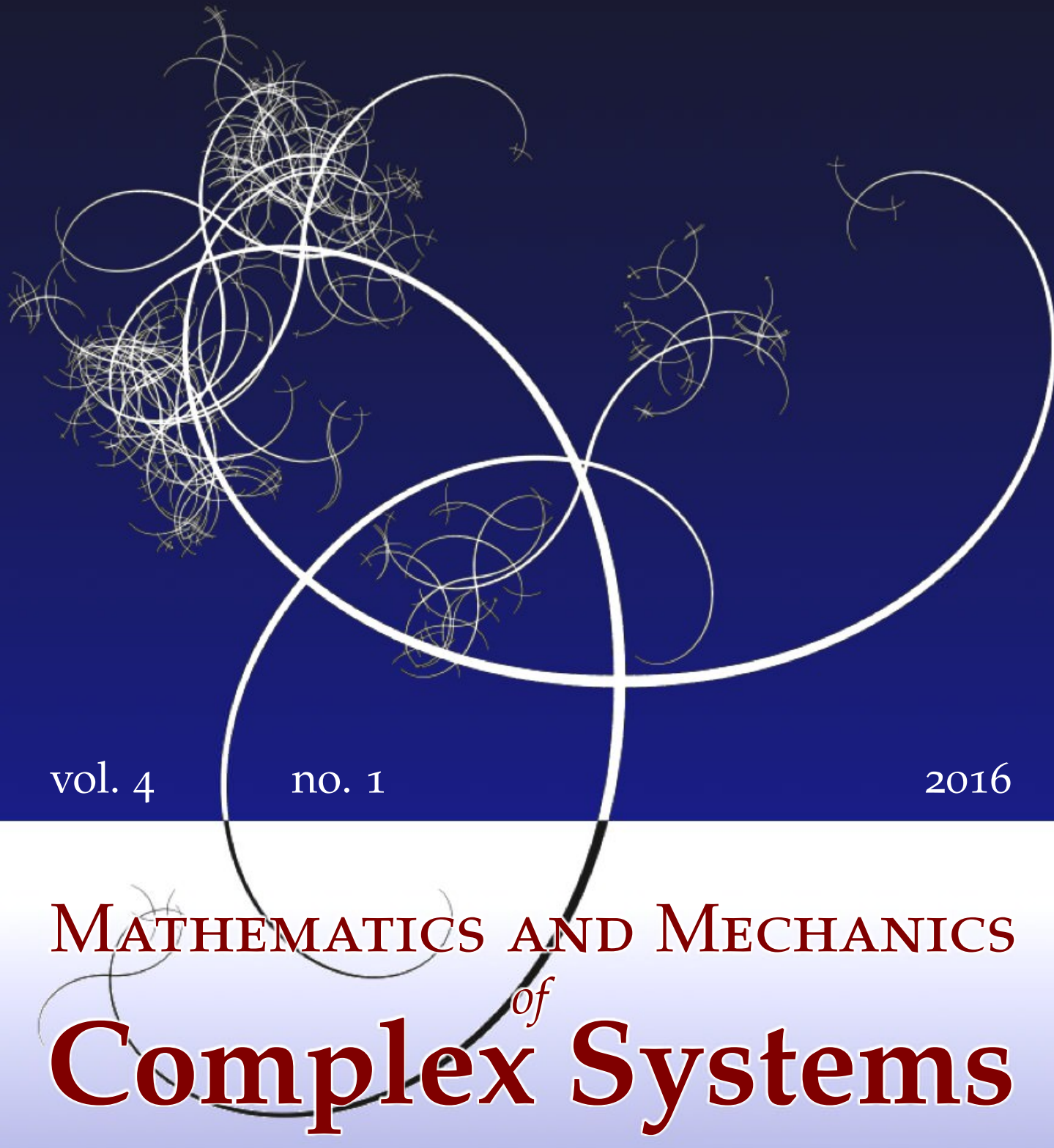

Pierluigi Vellucci and Alberto Maria Bersani ORTHOGONAL POLYNOMIALS AND RIESZ BASES APPLIED TO THE SOLUTION OF LOVE'S EQUATION 


\title{
ORTHOGONAL POLYNOMIALS AND RIESZ BASES APPLIED TO THE SOLUTION OF LOVE'S EQUATION
}

\author{
Pierluigi Vellucci AND Alberto Maria Bersani
}

\begin{abstract}
In this paper we reinvestigate the structure of the solution of a well-known Love's problem, related to the electrostatic field generated by two circular coaxial conducting disks, in terms of orthogonal polynomial expansions, enlightening the role of the recently introduced class of the Lucas-Lehmer polynomials. Moreover we show that the solution can be expanded more conveniently with respect to a Riesz basis obtained starting from Chebyshev polynomials.
\end{abstract}

\section{Introduction}

In 1949, E. R. Love [1949] considered the electrostatic field generated by two identical circular coaxial conducting disks at equal, and at equal and opposite, potentials, the potential at infinity being taken equal to zero. He established a celebrated expression for the potential, involving the solution of an integral equation of well-known type, much simpler than that considered by other authors in previous works.

Love's integral equation is a Fredholm equation of the second kind. It has found applications in several applied physics fields such as polymer structures, aerodynamics, fracture mechanics, hydrodynamics, and elasticity engineering. Recently, a polynomial expansion scheme was proposed by M. Agida and A. S. Kumar [2010] as an analytical method for solving Love's integral equation in the case of a rational kernel. Their study is concerned with the calculation of the normalized field created conjointly by two similar plates of radius $R$, separated by a distance $k R$, where $k$ is a positive real parameter, and at equal or opposite potential, with zero potential at infinity; the solution of this problem solves a Love's second kind integral equation; see also [Love 1990; Ren et al. 1999].

We propose two different approaches to this problem. In Section 2, starting from a classical technique, based on the expansion of the solution in orthogonal polynomials, we employ a class of polynomials introduced in [Vellucci and Bersani

\section{Communicated by Antonio Carcaterra.}

MSC2010: 00A69, 33C45.

Keywords: integral equations, numerical approximation and analysis, Love equation, Chebyshev polynomials, Lucas-Lehmer primality test, exponential bases, Riesz bases. 
2016], in order to solve a modified version of the original Love's equation. In Section 3, we recall a work by M. Norgren and B. L. G. Jonsson [2009], and we show that their results are still valid, expanding the solution of Love's integral equation with respect to a nonharmonic Fourier cosine series, which is a particular case of a Riesz basis [Sun and Zhou 1999].

For literature related to the numerical solutions of singular integral equations of the deterministic type, we refer to the fundamental book by L. Fox and I. B. Parker [1968], where different analytical methods for the solution of random integral equations were investigated.

\section{Chebyshev polynomials approach}

2.1. Preliminaries. In the following, we introduce the mathematical tools to employ an analytical method for solving Love's integral equation in the case of a rational kernel. Afterwards, we recall a short summary on Love's original problem.

2.1.1. Chebyshev and Lucas-Lehmer polynomials. The Chebyshev polynomials of the first kind [Chebyshev 1858; Chebyshev 1875; Erdélyi et al. 1953; Gatteschi 1973; Rivlin 1990] satisfy the recurrence relation

$$
\left\{\begin{array}{l}
T_{0}(x)=1 \\
T_{1}(x)=x \\
T_{n}(x)=2 x T_{n-1}(x)-T_{n-2}(x), \quad n \geq 2 .
\end{array}\right.
$$

The polynomials $T_{n}(x)$ are orthogonal with respect to the weight function $1 / \sqrt{1-x^{2}}$ defined on $x \in(-1,1)$. In a previous paper [Vellucci and Bersani 2016], we studied a class of polynomials $L_{n}(x)=L_{n-1}^{2}(x)-2$, created by means of the same iterative formula used to build the well-known Lucas-Lehmer sequence, employed in primality tests [Lucas 1878; Lehmer 1930; Ribenboim 1988; Bressoud 1989; Koshy 2001]. It is clearly crucial to choose the first term of the polynomial sequence. In [Vellucci and Bersani 2016], we showed that the Lucas-Lehmer polynomials are orthogonal in the interval $(-2,2)$ with respect to the weight $w(x)=1 /\left(4 \sqrt{4-x^{2}}\right)$ and such that their zeros belong to the interval $(-2,2)$, that is to their orthogonality interval. From now on we will consider $L_{0}=x$.

Let us first recall some important properties of these polynomials.

Proposition 1. For each $n \geq 1$,

$$
L_{n}(x)=2 T_{2^{n-1}}\left(\frac{1}{2} x^{2}-1\right)
$$

Proposition 2. The polynomials $L_{n}(x)$ are orthogonal with respect to the weight function $1 /\left(4 \sqrt{4-x^{2}}\right)$ defined on $x \in(-2,2)$. 
Corollary 3. With $x=2 \cos \theta$, the polynomials $L_{n}(x)$ admit the representation

$$
L_{n}(2 \cos \theta)=2 \cos \left(2^{n} \theta\right)
$$

For $|x| \leq 2$ we can show another formula for $L_{n}$ :

$$
L_{n}(x)=\left(\frac{1}{2} x^{2}-1-\sqrt{\left(\frac{1}{2} x^{2}-1\right)^{2}-1}\right)^{2^{n-1}}+\left(\frac{1}{2} x^{2}-1+\sqrt{\left(\frac{1}{2} x^{2}-1\right)^{2}-1}\right)^{2^{n-1}} .
$$

We change the sign inside the radical, factoring out the imaginary unit:

$$
L_{n}(x)=\left(\frac{1}{2} x^{2}-1-l \sqrt{1-\left(\frac{1}{2} x^{2}-1\right)^{2}}\right)^{2^{n-1}}+\left(\frac{1}{2} x^{2}-1+l \sqrt{1-\left(\frac{1}{2} x^{2}-1\right)^{2}}\right)^{2^{n-1}} .
$$

We then calculate the powers of the complex conjugate numbers $L_{n}^{+}$and $L_{n}^{-}$, depending on the variable $x$. Let

$$
\begin{aligned}
L_{n}(x) & =\left(\frac{1}{2} x^{2}-1+\sqrt{\left(\frac{1}{2} x^{2}-1\right)^{2}-1}\right)^{2^{n-1}}+\left(\frac{1}{2} x^{2}-1-\sqrt{\left(\frac{1}{2} x^{2}-1\right)^{2}-1}\right)^{2^{n-1}} \\
& =L_{n}^{+}(x)+L_{n}^{-}(x) .
\end{aligned}
$$

The absolute value of both complex numbers is unitary, since

$$
\begin{aligned}
& \left|L_{n}^{-}\right|=\left|\frac{1}{2} x^{2}-1-\imath \sqrt{1-\left(\frac{1}{2} x^{2}-1\right)^{2}}\right|^{2^{n-1},}, \\
& \left|L_{n}^{+}\right|=\left|\frac{1}{2} x^{2}-1+\imath \sqrt{1-\left(\frac{1}{2} x^{2}-1\right)^{2}}\right|^{2^{n-1}},
\end{aligned}
$$

and

$$
\left|L_{n}^{+}\right|=\left|L_{n}^{-}\right|=\sqrt{\left(\frac{1}{2} x^{2}-1\right)^{2}+1-\left(\frac{1}{2} x^{2}-1\right)^{2}}=1 .
$$

Moreover, since $L_{1}( \pm \sqrt{2})=0, L_{2}( \pm \sqrt{2})=-2$, and $L_{n}( \pm \sqrt{2})=2$ for all $n \geq 3$, the argument of $L_{n}( \pm \sqrt{2})$ is 0 for every $n \geq 3$. In the other cases, since we can write $x=2 \cos \vartheta$ when $|x| \leq 2$, it follows that $\frac{1}{2} x^{2}-1=\cos 2 \vartheta$. Thus for $|x| \neq \sqrt{2}$, we can also put

$$
\vartheta(x)=\frac{1}{2} \arctan \frac{\sqrt{1-\left(\frac{1}{2} x^{2}-1\right)^{2}}}{\frac{1}{2} x^{2}-1}+b \pi
$$

where $b$ is a binary digit. Finally, using (2), we obtain $L_{n}(x)=2 \cos \left(2^{n} \vartheta(x)\right)$.

By further setting

$$
\theta(x)=\frac{1}{2} \arctan \frac{\sqrt{1-\left(\frac{1}{2} x^{2}-1\right)^{2}}}{\frac{1}{2} x^{2}-1},
$$

we can write

$$
L_{n}(x)=2 \cos \left(2^{n} \theta(x)+2^{n} b \pi\right)=2 \cos \left(2^{n} \theta(x)\right) .
$$


On the other hand, the Chebyshev polynomials of the first kind can be defined as the unique polynomials satisfying

$$
T_{n}(t)=\cos (n \arccos t)
$$

or, in other words, as the unique polynomials satisfying

$$
T_{n}(\cos (\vartheta))=\cos (n \vartheta)
$$

for $n=0,1,2,3, \ldots$ Therefore, by Proposition 1 ,

$$
L_{n}(x)=2 T_{2^{n-1}}\left(\frac{1}{2} x^{2}-1\right)=2 \cos \left(2^{n-1} \arccos \left(\frac{1}{2} x^{2}-1\right)\right) .
$$

2.1.2. Love's problem. Two leading cases of the problem are considered here: to specify the field generated by two identical circular coaxial conducting disks (a) at equal potentials and (b) at equal and opposite potentials, the potential at infinity being taken as zero. The results established by Love are as follows: the upper sign referring to the case of equally charged disks and the lower to that of oppositely charged disks. For Theorem 4 we refer to [Love 1949, Figures 1 and 2].

Theorem 4 [Love 1949]. In the two leading cases described above, the potential at any point $\left(p, \zeta, \zeta^{\prime}\right)$, specified by its distance $r=\rho$ a from the axis of the disks and its axial distances $z=\zeta$ a and $z^{\prime}=\zeta^{\prime}$ a from their planes, is

$$
\frac{V_{0}}{\pi} \int_{-1}^{1}\left(\frac{1}{\sqrt{\rho^{2}+(\zeta+i t)^{2}}} \pm \frac{1}{\sqrt{\rho^{2}+\left(\zeta^{\prime}+i t\right)^{2}}}\right) f(t) d t
$$

where $V_{0}$ is potential of the disks, a is the radius of the disks, each square root has positive real part, and $f(t)$ is the solution of the integral equation

$$
f(x) \pm \frac{1}{\pi} \int_{-1}^{1} \frac{k}{k^{2}+(x-t)^{2}} f(t) d t=1, \quad|x| \leq 1
$$

where $k$ is the spacing parameter.

Theorem 5 [Love 1949]. For every positive $k$, (11) has a continuous solution, and no other solution: it is real and even, and is specifiable by the Neumann series

$$
f(x)=1+\sum_{n=1}^{\infty}(\mp 1)^{n} \int_{-1}^{1} K_{n}(x, t) d t,
$$

where the iterated kernels $K_{n}(x, t)$, for $n \in \mathbb{N}, n>1$, are given by

$$
K_{1}(x, t)=\frac{1}{\pi} \frac{k}{k^{2}+(x-t)^{2}} \quad \text { and } \quad K_{n}(x, t)=\int_{-1}^{1} K_{n-1}(x, s) K_{1}(s, t) d s .
$$


Theorem 6 [Love 1949]. The capacitance of each disk in the two cases is

$$
\frac{a}{\pi} \int_{-1}^{1} f(t) d t
$$

and the components of the field at all points not on the disks are given by the appropriate formal differentiations of (10).

\subsection{The classical approach to the problem in terms of orthogonal polynomials.}

For the solution of the problems we will refer to [Fox and Parker 1968]. When the upper and lower disks are at potentials $V_{0}$ and $\pm V_{0}$, the potential $V$ at any point whose spheroidal coordinates are $(\mu, \eta)$ with respect to the upper disk and $\left(\mu^{\prime}, \eta^{\prime}\right)$ with respect to the lower one is expressed in terms of Legendre functions. The upper disk, specified in cylindrical polar coordinates $(r, \theta, z)$ by $r \leq a$ and $z=0$, is taken as "focal disk" $\eta=0$ of spheroidal coordinates $(\mu, \eta)$; in actual study these are such that $-2 \leq \mu \leq 2, \eta \geq 0$.

Then (10) can be rewritten in the form

$$
\frac{V_{0}}{2 \pi} \int_{-2}^{2}\left(\frac{1}{\sqrt{\rho^{2}+(\zeta+i t / 2)^{2}}} \pm \frac{1}{\sqrt{\rho^{2}+\left(\zeta^{\prime}+i t / 2\right)^{2}}}\right) f(t / 2) d t
$$

where each square root has positive real part, and $f(t)$ is the solution of the integral equation

$$
f(x) \pm \frac{1}{2 \pi} \int_{-2}^{2} \frac{k}{k^{2}+(x-t / 2)^{2}} f(t / 2) d t=1, \quad|x| \leq 2
$$

By the linear transformation $t=2 y$, both equations can be reduced to Love's original form. In (14) we put $k=1$ and consider positive sign, so

$$
f(x)+\frac{1}{2 \pi} \int_{-2}^{2} \frac{1}{1+(x-t / 2)^{2}} f(t / 2) d t=1, \quad|x| \leq 2 .
$$

We make the substitution $x \mapsto \frac{1}{2} x^{2}-1$ in (15), yielding

$$
f\left(\frac{1}{2} x^{2}-1\right)+\frac{1}{2 \pi} \int_{-2}^{2} \frac{1}{1+\left(\frac{1}{2}\left(x^{2}-t\right)-1\right)^{2}} f\left(\frac{1}{2} t\right) d t=1 .
$$

We can find a Chebyshev series solution as follows: write

$$
f(x)=\sum_{r=0}^{\infty} a_{r} T_{r}(x)
$$


substitute it into (15), interchange the order of integration and summation in the first term. Then we arrive at the equation

$$
\sum_{r=0}^{\infty} a_{r} T_{r}\left(\frac{1}{2} x^{2}-1\right)+\frac{1}{2 \pi} \sum_{s=0}^{\infty} a_{s} \int_{-2}^{2} \frac{T_{s}\left(\frac{1}{2} t\right)}{1+\left(\frac{1}{2}\left(x^{2}-t\right)-1\right)^{2}} d t=1
$$

for $|x| \leq 2$. If we can now determine the expansion

$$
\frac{1}{2} \int_{-2}^{2} \frac{T_{s}\left(\frac{1}{2} t\right)}{1+\left(\frac{1}{2}\left(x^{2}-t\right)-1\right)^{2}} d t=\sum_{r=0}^{\infty} b_{s r} T_{r}\left(\frac{1}{2} x^{2}-1\right),
$$

we can equate the corresponding coefficients of each $T_{r}(x)$ on both sides of (15), which is legitimate since the Chebyshev polynomials form a complete set of independent functions, to produce an infinite set of algebraic equations for the required coefficients $a_{r}$, given by

$$
a_{r}+\sum_{s=0}^{\infty} a_{s} b_{s r}=0, \quad r=1,2, \ldots
$$

and, for $r=0$,

$$
a_{0}+\sum_{s=0}^{\infty} a_{s} b_{s, 0}=1 .
$$

The $a_{r}$ will decrease rapidly for sufficiently large $r$, so that in a convenient method of solving (17) we select the first $n+1$ rows and columns, perform Gaussian elimination and back-substitution for the last few coefficients $-a_{n}, a_{n-1}, a_{n-2}$, say decide by inspection whether convergence is sufficiently rapid for the required precision with this selected value of $n$, and if necessary add some extra rows and columns with only a small additional amount of work.

Let's go back to (16). Let

$$
J=\{1,2,4, \ldots\}=\left\{2^{r-1} \mid r \in \mathbb{N}\right\},
$$

and rewrite (16) in this way:

$$
\sum_{r=0}^{\infty} a_{r} T_{r}\left(\frac{1}{2} x^{2}-1\right)+\sum_{s=0}^{\infty} a_{s} \sum_{r=0}^{\infty} c_{s r} T_{r}\left(\frac{1}{2} x^{2}-1\right)=1,
$$

where $c_{s r}=b_{s r} / \pi$. Then

$$
\begin{aligned}
\sum_{r \in J} a_{r} T_{r}\left(\frac{1}{2} x^{2}-1\right)+\sum_{r \notin J} a_{r} T_{r}\left(\frac{1}{2} x^{2}-1\right) & \\
& +\sum_{s=0}^{\infty} a_{s}\left(\sum_{r \in J} c_{s r} T_{r}\left(\frac{1}{2} x^{2}-1\right)+\sum_{r \notin J} c_{s r} T_{r}\left(\frac{1}{2} x^{2}-1\right)\right)=1 .
\end{aligned}
$$


By (1),

$$
\begin{aligned}
\frac{1}{2} \sum_{r=1}^{\infty} a_{r} L_{r}(x)+\sum_{r \notin J} a_{r} T_{r}\left(\frac{1}{2} x^{2}-1\right) & \\
& +\frac{1}{2} \sum_{s=0}^{\infty} a_{s} \sum_{r=1}^{\infty} c_{s r} L_{r}(x)+\sum_{s=0}^{\infty} a_{s} \sum_{r \notin J} c_{s r} T_{r}\left(\frac{1}{2} x^{2}-1\right)=1
\end{aligned}
$$

By Proposition 1, we note that solving (17), a subset of first $n+1$ rows and columns selected to perform Gaussian elimination, is due to Lucas-Lehmer polynomials. They not only cannot by themselves guarantee the convergence to the solution, but also their contributions can be neglected. In fact, by above reasoning, since

$$
f(x)=\sum_{r \notin J} a_{r} T_{r}\left(\frac{1}{2} x^{2}-1\right)+\frac{1}{2} \sum_{r \in J} a_{r} L_{r}(x),
$$

we have

$$
\left|f(x)-\sum_{r \notin J} a_{r} T_{r}\left(\frac{1}{2} x^{2}-1\right)\right| \leq \frac{1}{2} \sum_{r \in J}\left|a_{r}\right|=\frac{1}{2} \sum_{r=1}^{\infty}\left|a_{2^{r-1}}\right| .
$$

Accordingly, when the term on the right hand side can be considered "small" with respect to other contributions, a convenient method of solving (17) should be to select the first $n+1$ rows and columns, perform Gaussian elimination and backsubstitution for the last few coefficients $-a_{n}, a_{n-1}, a_{n-2}$, say - and delete terms due to Lucas-Lehmer polynomials.

\section{An alternative approach: nonharmonic Fourier series}

The capacitance of a circular parallel plate capacitor can be calculated by expanding the solution of the Love's integral equation in terms of a Fourier cosine series. In previous literature, this kind of expansion was carried out numerically, leading to accuracy problems at small plate separations. Norgren and Jonsson [2009] calculated analytically all expansion integrals in terms of the sine and cosine integrals. Hence, they approximated the kernel using considerably large matrices, resulting in improved numerical accuracy for the capacitance. Previously, G. T. Carlson and B. L. Illman [1994], solved the Love's equation through an expansion of the kernel into a Fourier cosine series. To calculate the expansion coefficients of the kernel, they use numerical integration. Hence, as noted in [Norgren and Jonsson 2009], their method is limited by a combination of the accuracy of the integration and the large number of terms needed. The accumulated errors effectively limit the expansion to about 100 terms, which is insufficient for the convergence at very small separations. Let us observe that both the methods recalled here make use of orthogonal expansions. 
In this section we will use some basic facts about nonharmonic Fourier series, and we recall them below.

It is well known that the family of exponentials $\left\{e^{i n t}\right\}_{n \in \mathbb{Z}}$ forms an orthonormal basis in $L^{2}(-\pi, \pi)$. The natural question that arises is: what happens if we replace it by a classical system of exponentials $\left\{e^{i \lambda_{n} t}\right\}_{n \in \mathbb{Z}}$; these bases are very useful for the study of the so-called almost periodic functions. See, for example, [Andres et al. 2006; Besicovitch 1932].

The celebrated work of Paley and Wiener [1934] kicked off studies on classical systems of exponentials $\left\{e^{i \lambda_{n} t}\right\}_{n \in \mathbb{Z}}$ in $L^{2}(0, T)$, where $T>0$. They proved that if $\lambda_{n} \in \mathbb{R}, n \in \mathbb{Z}$, and

$$
\left|\lambda_{n}-n\right| \leq L<\pi^{-2}, \quad n \in \mathbb{Z}
$$

then the system $\left\{e^{i \lambda_{n} t}\right\}_{n \in \mathbb{Z}}$ forms a Riesz basis in $L^{2}[-\pi, \pi]$, i.e., a family of the form $\left\{U e_{k}\right\}_{k=1}^{\infty}$, where $\left\{e_{k}\right\}_{k=1}^{\infty}$ is an orthonormal basis for a separable infinitedimensional Hilbert space $\mathscr{H}$ and $U: \mathscr{H} \rightarrow \mathscr{H}$ is a bounded bijective operator.

M. I. Kadec [1964] extended this result to the case $L<\frac{1}{4}$. This is the socalled Kadec- $\frac{1}{4}$ theorem, which over the following 50 years has been extensively generalized; see, for example [Avdonin 1974; Bailey 2010; Pavlov 1979; Sedletskii 2003; Sun and Zhou 1999; Vellucci 2015]. Let us recall Kadec's original result:

Theorem 7. If $\left\{\lambda_{n}\right\}_{n \in \mathbb{Z}}$ is a sequence of real numbers for which

$$
\sup _{n}\left|\lambda_{n}-n\right|<\frac{1}{4}, \quad n=0, \pm 1, \ldots
$$

then the system $\left\{e^{i \lambda_{n} t}\right\}_{n \in \mathbb{Z}}$ is a Riesz basis for $L^{2}[-\pi, \pi]$.

Therefore, if $L=\sup _{n}\left|\lambda_{n}-n\right|<\frac{1}{4}$, then the sine system $\left\{\sin \lambda_{n} t\right\}_{1}^{\infty}$ as well as the cosine system $1 \cup\left\{\cos \lambda_{n} t\right\}_{1}^{\infty}$ is a Riesz basis in $L^{2}(0, \pi)$.

We now approach the problem described in [Norgren and Jonsson 2009]. The circular parallel plate capacitor is depicted in Figure 1.

The distance between the circular plates is here put equal to their common radius. Accordingly, the normalized separation between the plates, a constant $k$, is set for

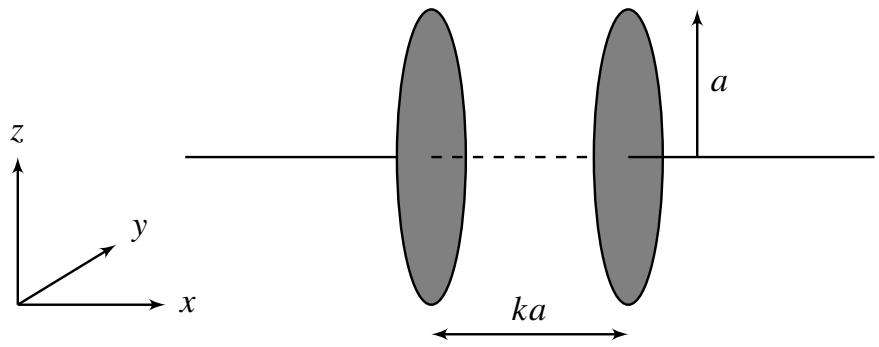

Figure 1. A circular parallel plate capacitor can be viewed as a cylindrical volume whose bases are the capacitor's plates. 
the sake of simplicity equal to 1 . The model is idealized in the sense that the plates have zero thickness.

The capacitance of the parallel plate capacitor is [Carlos and Illman 1994]

$$
C=4 \varepsilon_{0} a \int_{0}^{1} f(s) d s,
$$

where $a$ is the radius of the circular plate and the function $f(s)$ is the solution of the modified Love's integral equation

$$
f(s)-\int_{0}^{1} K(s, t) f(t) d t=1, \quad 0 \leq s \leq 1,
$$

with kernel

$$
K(s, t)=\frac{1}{\pi}\left(\frac{1}{1+(s-t)^{2}}+\frac{1}{1+(s+t)^{2}}\right) .
$$

To solve (19) numerically, we follow the approach in [loc. cit.] and expand the kernel and the unknown function into the (nonharmonic) Fourier cosine expansion in terms of the functions

$$
\tilde{\psi}_{n}(s)=\sqrt{2-\delta_{n, 0}} \cos \left(\lambda_{n} s\right), \quad n=0,1, \ldots
$$

which in our study have been orthonormalized to fulfill the orthogonality relation

$$
\int_{0}^{\pi} \psi_{n}(s) \psi_{m}(s) d s=\delta_{m, n}
$$

and satisfy Kadec's assumption on $L=\sup _{n}\left|\lambda_{n}-n\right|<\frac{1}{4}$. Here, $\delta_{m, n}$ denotes the Kronecker delta function.

This orthonormalization process is shown in the following

Theorem 8 (orthonormalization process). Consider $L^{2}(-\pi, \pi)$ and a sequence $\left\{\lambda_{n}\right\}_{n \in \mathbb{Z}} \subset \mathbb{R}$ which satisfies Kadec's assumption. Let $P=(I-S)^{-1}=\sum_{m=0}^{\infty} S^{m}$, where

$$
S(f)(x)=\sum_{n=-\infty}^{\infty} \hat{f}(n)\left(e^{i n x}-e^{i \lambda_{n} x}\right)
$$

and $\{\hat{f}(n)\}$ are the Fourier coefficients of $f$. Then $P\left(e^{i \lambda_{n} x}\right)=e^{i n x}$ for each $n \in \mathbb{Z}$. Proof. By Kadec's theorem, we have that $\|S\|<1$. Hence, $P=(I-S)^{-1}=$ $\sum_{m=0}^{\infty} S^{m}$. To show that $P\left(e^{i \lambda_{n} x}\right)=e^{i n x}$, we write

$$
e^{i \lambda_{n} x}=(I-S) e^{i n x}=e^{i n x}-\sum_{k} c_{k}\left(e^{i k x}-e^{i \lambda_{k} x}\right)
$$

where $c_{k}=\left\langle e^{i n x}, e^{i k x}\right\rangle$. Thus

$$
e^{i n x}-e^{i \lambda_{n} x}=\sum_{k} \delta_{n, k}\left(e^{i k x}-e^{i \lambda_{k} x}\right) .
$$


In this way we have orthonormalized the Riesz basis $\left\{e^{i \lambda_{n} x}\right\}$, in an easy way. Further results on the orthonormalization of more complex Riesz bases, such as $\{\phi(t-n)\}_{n \in \mathbb{Z}}$, applied for example to the study of a "digital filter," can be found in [Meyer 1989]. For our purposes it is sufficient to consider the basis introduced at the beginning of Section 3 and used in Theorem 7.

Carrying out the expansions of $f(s)$ and $K(s, t)$ in terms of $\left\{\psi_{n}\right\}$, we obtain

$$
f(s)=\sum_{m=0}^{\infty} f_{m} \psi_{m}(s), \quad \text { where } f_{m}=\int_{0}^{\pi} f(s) \psi_{m}(s) d s
$$

and

$$
K(s, t)=\sum_{m=0}^{\infty} \sum_{n=0}^{\infty} K_{m n} \psi_{m}(s) \psi_{n}(t),
$$

where

$$
K_{m n}=\int_{0}^{\pi} \int_{0}^{\pi} K(s, t) \psi_{n}(t) \psi_{m}(s) d s d t .
$$

These equations yield the infinite linear system of equations for the coefficients $\left\{f_{n}\right\}_{n=0}^{\infty}$ :

$$
\sum_{n=0}^{\infty}\left(\delta_{m, n}-K_{m n}\right) f_{n}=\delta_{m, 0}, \quad m=0,1, \ldots
$$

From (18), (21), and from the orthonormalization process described in Theorem 8 and guaranteed by Kadec's assumption, which allows to us expand the kernel and the unknown function into the (nonharmonic) Fourier cosine expansion in terms of the functions $\left\{\cos \left(\lambda_{n} s\right)\right\}$, the capacitance reduces to $C=4 \varepsilon_{0} a f_{0}$, where $f_{0}$ is simply the $(0,0)$-element in the inverse of the matrix with elements $\delta_{m, n}-K_{m n}$, as obtained in [Norgren and Jonsson 2009].

Furthermore, Norgren and Jonsson derive analytical expressions for the expansion of the kernel $K(s, t)$. Proceeding as in [loc. cit.], it is easy to prove that, in the general case when $m \neq n$ and $m, n>0$,

$$
K_{m n}=\frac{2}{\pi} \tilde{I}_{3}(n \pi, m \pi),
$$

where $\tilde{I}_{3}(n \pi, m \pi)=P I_{3}\left(\lambda_{n} \pi, \lambda_{m} \pi\right)$, with $P$ as in Theorem 8 and $I_{3}$ as defined in [loc. cit.]. The application of the operator $P$ denotes here the orthonormalization process performed on the set of functions $\left\{\cos \left(\lambda_{n} s\right)\right\}_{n \in \mathbb{Z}}$.

We have extended the results of [Carlos and Illman 1994; Norgren and Jonsson 2009] to a (nonharmonic) Fourier cosine expansion in terms of the set of functions $\left\{\cos \left(\lambda_{n} s\right)\right\}_{n \in \mathbb{Z}}$, employing a simple procedure, due to Theorem 8 , to orthonormalize the Riesz basis $\left\{e^{i \lambda_{n} x}\right\}$ under Kadec's assumption. Therefore, we have found a further expansion of the solution that it is not in terms of orthogonal polynomials, but in terms of nonharmonic functions $\cos \left(\lambda_{n} s\right), s \in \mathbb{R}$. 


\section{Conclusion and perspectives}

Orthogonal functions, other classes of polynomials, and Riesz bases have shown to be very powerful for the search of solutions of several problems in disparate fields, from physics to engineering, from economics to biology, and so on. In this paper we applied a new class of orthogonal polynomials, called Lucas-Lehmer polynomials [Vellucci and Bersani 2016] and the tool of Riesz bases [Paley and Wiener 1934] in order to reinvestigate a classical problem, due to Love [1949], obtaining a further expansion of the solution that it is not in terms of orthogonal polynomials, but in terms of nonharmonic functions $\cos \left(\lambda_{n} s\right), s \in \mathbb{R}$, suitably orthonormalized, thanks to Theorem 8 which uses the celebrated result due to Kadec [1964]. Many other applications can be investigated in the future, mainly in the field of mechanics. In particular, great attention has been recently paid to peridynamics and fracture mechanics, which are approached in terms of integral equations. In this framework our researches will be addressed to apply our techniques to the study of some integral equations of the type introduced by Piola in 1848, recently rediscovered in the framework of peridynamics and fracture dynamics, and reported in the paper [dell'Isola et al. 2015].

\section{References}

[Agida and Kumar 2010] M. Agida and A. S. Kumar, "A Boubaker polynomials expansion scheme solution to random Love's equation in the case of a rational kernel", Electron. J. Theor. Phys. 7:24 (2010), 319-326.

[Andres et al. 2006] J. Andres, A. M. Bersani, and R. F. Grande, "Hierarchy of almost-periodic function spaces", Rend. Mat. Appl. (7) 26:2 (2006), 121-188.

[Avdonin 1974] S. A. Avdonin, "On the question of Riesz bases of exponential functions in $L^{2}$ ", Vestnik Leningrad. Univ. 13 (1974), 5-12. In Russian.

[Bailey 2010] B. Bailey, "Sampling and recovery of multidimensional bandlimited functions via frames", J. Math. Anal. Appl. 367:2 (2010), 374-388.

[Besicovitch 1932] A. S. Besicovitch, Almost periodic functions, Cambridge University Press, 1932. [Bressoud 1989] D. M. Bressoud, Factorization and primality testing, Springer, New York, 1989.

[Carlos and Illman 1994] G. T. Carlos and B. L. Illman, "The circular disk parallel plate capacitor", Amer. J. Phys. 62:12 (1994), 1099-1105.

[Chebyshev 1858] P. L. Chebyshev, "Sur une nouvelle série”, Bull. Acad. sci. St. Pétersbourg 17 (1858). Reprinted as pp. 379-384 in Euvres de P. L. Tchebychef, vol. I, St. Petersburg, 1899.

[Chebyshev 1875] P. L. Chebyshev, “Объ интерполированıи величинъ равноотцсоящихъ”, Zap. Imp. Akad. Nauk (St. Petersburg) 25:5 (1875). Translated as "Sur l'interpolation des valeurs équidistantes", on pp. 219-242 in Euvres de P. L. Tchebychef, vol. II, St. Petersburg, 1899.

[dell'Isola et al. 2015] F. dell'Isola, U. Andreaus, and L. Placidi, "At the origins and in the vanguard of peridynamics, non-local and higher-gradient continuum mechanics: an underestimated and still topical contribution of Gabrio Piola", Math. Mech. Solids 20:8 (2015), 887-928.

[Erdélyi et al. 1953] A. Erdélyi, W. Magnus, F. Oberhettinger, and F. G. Tricomi, Higher transcendental functions, vol. II, McGraw-Hill, New York-Toronto-London, 1953. 
[Fox and Parker 1968] L. Fox and I. B. Parker, Chebyshev polynomials in numerical analysis, Oxford University Press, London-New York-Toronto, 1968.

[Gatteschi 1973] L. Gatteschi, Funzioni speciali, Unione Tipografico-Editrice Torinese, 1973.

[Kadec 1964] M. Ĭ. Kadec, “The exact value of the Paley-Wiener constant”, Dokl. Akad. Nauk SSSR 155 (1964), 1253-1254. In Russian; translated in Sov. Math. 5 (1964), 559-561.

[Koshy 2001] T. Koshy, Fibonacci and Lucas numbers with applications, Wiley, New York, 2001.

[Lehmer 1930] D. H. Lehmer, "An extended theory of Lucas' functions", Ann. of Math. (2) 31:3 (1930), 419-448.

[Love 1949] E. R. Love, "The electrostatic field of two equal circular co-axial conducting disks", Quart. J. Mech. Appl. Math. 2 (1949), 428-451.

[Love 1990] E. R. Love, "The potential due to a circular parallel plate condenser", Mathematika 37:2 (1990), 217-231.

[Lucas 1878] E. Lucas, “Theorie des Fonctions Numeriques Simplement Periodiques", Amer. J. Math. 1:4 (1878), 289-321.

[Meyer 1989] Y. Meyer, "Wavelets and operators", pp. 256-365 in Analysis at Urbana (Urbana, IL, 1986-87), vol. 1, edited by E. Berkson and T. Peck, London Math. Soc. Lecture Note Ser. 137, Cambridge Univ. Press, 1989.

[Norgren and Jonsson 2009] M. Norgren and B. L. G. Jonsson, "The capacitance of the circular parallel plate capacitor obtained by solving the Love integral equation using an analytic expansion of the kernel", Progr. Electromag. Res. 97 (2009), 357-372.

[Paley and Wiener 1934] R. E. A. C. Paley and N. Wiener, Fourier transforms in the complex domain, Amer. Math. Soc. Colloq. Publ. 19, American Mathematical Society, New York, 1934.

[Pavlov 1979] B. S. Pavlov, "The basis property of a system of exponentials and the condition of Muckenhoupt", Dokl. Akad. Nauk SSSR 247:1 (1979), 37-40. In Russian; translated in Sov. Math. 20 (1979), 655-659.

[Ren et al. 1999] Y. Ren, B. Zhang, and H. Qiao, "A simple Taylor-series expansion method for a class of second kind integral equations”, J. Comput. Appl. Math. 110:1 (1999), 15-24.

[Ribenboim 1988] P. Ribenboim, The book of prime number records, Springer, New York, 1988.

[Rivlin 1990] T. J. Rivlin, Chebyshev polynomials, 2nd ed., Wiley, New York, 1990.

[Sedletskii 2003] A. M. Sedletskii, "Nonharmonic analysis", J. Math. Sci. (New York) 116:5 (2003), 3551-3619.

[Sun and Zhou 1999] W. Sun and X. Zhou, "On the stability of multivariate trigonometric systems", J. Math. Anal. Appl. 235:1 (1999), 159-167.

[Vellucci 2015] P. Vellucci, "A simple pointview for Kadec-1/4 theorem in the complex case", Ric. Mat. 64:1 (2015), 87-92.

[Vellucci and Bersani 2016] P. Vellucci and A. M. Bersani, "The class of Lucas-Lehmer polynomials", (2016). To appear in Rend. Mat. Appl. (7). arXiv 1603.01989

Received 15 Oct 2015. Revised 8 Dec 2015. Accepted 6 Feb 2016.

PIERLUIGI VelluCCI: pierluigi.vellucci@sbai.uniroma1.it

Dipartimento di Scienze di Base e Applicate per l'Ingegneria, Sapienza Università di Roma, Via Antonio Scarpa 16, I-00161 Rome, Italy

Alberto MARIA Bersani: alberto.bersani@sbai.uniroma1.it

Dipartimento di Scienze di Base e Applicate per l'Ingegneria, Sapienza Università di Roma, Via Antonio Scarpa 16, I-00161 Rome, Italy 
EDITORIAL BOARD

ANTONIO CARCATERRA

ERIC A. CARLEN

FRANCESCO DELL'ISOLA

RAFFAELE ESPOSITO

ALBERT FANNJIANG

Gilles A. FranCFORT

Pierangelo MARCATI

JEAN-JACQUES MARIGO

PETER A. MARKOWICH

MARTIN OSTOJA-STARZEWSKI

PIERRE SEPPECHER

DAVID J. STEIGMANN

PAUl STEINMANN

PierRe M. SuQueT

MANAGING EDITORS

MICOL AMAR

CORRADO LATTANZIO

ANGELA MADEO

MARTIN OSTOJA-STARZEWSKI

ADVISORY BOARD

ADNAN AKAY

Holm AltenBaCH

MICOL AMAR

HARM ASKES

TEODOR ATANACKOVIĆ

VICTOR BERDICHEVSKY

GUY BOUCHITTÉ

ANDREA BRAIDES

ROBERTO CAMASSA

MAURO CARFORE

ERIC DARVE

FELIX DARVE

ANNA DE MASI

GianPiEtro DEL Piero

EMMANUELE Di BENEDETTO

BERNOLD FIEDLER

IRENE M. GAMBA

DAVID Y. GAO

SERGEY GAVRILYUK

TIMOTHY J. HEALEY

DOMINIQUE JEULIN

ROGER E. KHAYAT

CORRADO LATTANZIO

ROBERT P. LIPTON

ANGELO LUONGO

ANGELA MADEO

JUAN J. MANFREDI

CARLO MARCHIORO

GÉRARD A. MAUGIN

ROBERTO NATALINI PATRIZIO NEFF

ANDREY PIATNITSKI

ERRICO PRESUTTI

MARIO PULVIRENTI

LUCIO RUSSO

Miguel A. F. SANJUAN

PATRICK SElVADURAI

ALEXANDER P. SEYRANIAN

MIROSLAV ŠILHAVÝ

GUIDO SWEERS

ANTOINETTE TORDESILLAS

LEV TRUSKINOVSKY

JUAN J. L. VELÁZQUEZ VINCENZO VESPRI ANGELO VULPIANI msp.org/memocs

Università di Roma "La Sapienza", Italia

Rutgers University, USA

(CO-CHAIR) Università di Roma "La Sapienza", Italia

(TREASURER) Università dell'Aquila, Italia

University of California at Davis, USA

(CO-CHAIR) Université Paris-Nord, France

Università dell'Aquila, Italy

École Polytechnique, France

DAMTP Cambridge, UK, and University of Vienna, Austria

(CHAIR MANAGING EDITOR) Univ. of Illinois at Urbana-Champaign, USA

Université du Sud Toulon-Var, France

University of California at Berkeley, USA

Universität Erlangen-Nürnberg, Germany

LMA CNRS Marseille, France

Università di Roma "La Sapienza", Italia

Università dell'Aquila, Italy

Université de Lyon-INSA (Institut National des Sciences Appliquées), France

(CHAIR MANAGING EDITOR) Univ. of Illinois at Urbana-Champaign, USA

Carnegie Mellon University, USA, and Bilkent University, Turkey

Otto-von-Guericke-Universität Magdeburg, Germany

Università di Roma "La Sapienza", Italia

University of Sheffield, UK

University of Novi Sad, Serbia

Wayne State University, USA

Université du Sud Toulon-Var, France

Università di Roma Tor Vergata, Italia

University of North Carolina at Chapel Hill, USA

Università di Pavia, Italia

Stanford University, USA

Institut Polytechnique de Grenoble, France

Università dell'Aquila, Italia

Università di Ferrara and International Research Center MEMOCS, Italia

Vanderbilt University, USA

Freie Universität Berlin, Germany

University of Texas at Austin, USA

Federation University and Australian National University, Australia

Université Aix-Marseille, France

Cornell University, USA

École des Mines, France

University of Western Ontario, Canada

Università dell' Aquila, Italy

Louisiana State University, USA

Università dell'Aquila, Italia

Université de Lyon-INSA (Institut National des Sciences Appliquées), France University of Pittsburgh, USA

Università di Roma "La Sapienza”, Italia

Université Paris VI, France

Istituto per le Applicazioni del Calcolo "M. Picone", Italy

Universität Duisburg-Essen, Germany

Narvik University College, Norway, Russia

Università di Roma Tor Vergata, Italy

Università di Roma "La Sapienza”, Italia

Università di Roma “Tor Vergata”, Italia

Universidad Rey Juan Carlos, Madrid, Spain

McGill University, Canada

Moscow State Lomonosov University, Russia

Academy of Sciences of the Czech Republic

Universität zu Köln, Germany

University of Melbourne, Australia

École Polytechnique, France

Bonn University, Germany

Università di Firenze, Italia

Università di Roma La Sapienza, Italia

MEMOCS (ISSN 2325-3444 electronic, 2326-7186 printed) is a journal of the International Research Center for the Mathematics and Mechanics of Complex Systems at the Università dell'Aquila, Italy.

Cover image: "Tangle” by $\odot$ John Horigan; produced using the Context Free program (contextfreeart.org).

PUBLISHED BY

7 mathematical sciences publishers

nonprofit scientific publishing

http://msp.org/

(C) 2016 Mathematical Sciences Publishers 
Mathematics and Mechanics of Complex Systems vol. 4 no. 1

Gradient materials with internal constraints

Albrecht Bertram and Rainer Glüge

Unified geometric formulation of material uniformity and evolution

Marcelo Epstein and Manuel de León

Electromechanics of polarized lipid bilayers

David J. Steigmann and Ashutosh Agrawal

Orthogonal polynomials and Riesz bases applied to the solution of Love's equation

Pierluigi Vellucci and Alberto Maria Bersani

Modeling capillary hysteresis in unsatured porous media

Gérard Gagneux and Olivier Millet

Discrete double-porosity models for spin systems

Andrea Braides, Valeria Chiadò Piat and Margherita Solci

Correction to "On the theory of diffusion and swelling in finitely deforming elastomers"

Gary J. Templet and David J. Steigmann

MEMOCS is a journal of the International Research Center for the Mathematics and Mechanics of Complex Systems at the Università dell' Aquila, Italy.

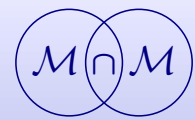

